Conclusion In the group of massaged infant seems to obtain better results in motor milestones and in cognitive development, faster maturation of stereopsis, a increase of visual acuity have been also reached.

\section{EPIDEMIOLOGICAL ASPECTS OF STREPTOCOCCAL PHARYNGEAL INFECTIONS IN PEDIATRIC POPULATION}

doi:10.1136/archdischild-2012-302724.1737

'S lurian, ${ }^{2} \mathrm{~L}$ Bera, ${ }^{3} \mathrm{SI}$ lurian, ${ }^{1} \mathrm{M}$ Mihut, ${ }^{3} \mathrm{ML}$ Neamtu, ${ }^{4} \mathrm{~A}$ Muntean. ${ }^{1}$ Clinical Laboratory, Pediatric Hospital; '2Statistics Department, Faculty of Medicine, 'Lucian Blaga' University; ${ }^{3}$ Research Department, Pediatric Clinic, 'Lucian Blaga' University; ${ }^{4}$ Pediatric Clinic, Sibiu, Romania

Background During last 5 years, we noticed an increasing incidence of scarlet fever and streptococcal pharyngitis in our county. Aims

1. To appreciate the positive results rate for beta-hemolytic pyogenic streptococci in throat specimens (group A streptococci- gAs, group C- gCs, group G- gGs);

2. To evaluate ratio of each streptococcal group pharyngeal infection;

3. To establish correlation between streptococcal infections and diseases that justified throat cultures.

Methods Authors designed a retrospective epidemiological study, analyzing microbiology department data during 14 months period. Inclusion criteria: hospitalized and ambulatory care children aged between 2-18 years (scarlet fever diagnosis, pharyngitis diagnosis, healthy children requesting throat exam). Exclusion criteria: children up to 2 years of age. In order to identify streptococci, authors used Columbia agar with $5 \%$ sheep blood, Bacitracin inhibition tests, latex agglutination. Data was statistically analyzed using likelihood ratio.

Results Among 6653 throat cultures, 497 isolates (7.47\%) were positive. Ratio for each streptococci group was: group A $-88.0 \%$, group C $-6.2 \%$, group G $-5.8 \%$. Seasonal incidence: higher incidence was reported in February and lower incidence in August. Regarding correlation between patient diagnosis and identified streptococci group in throat specimens ( $\mathrm{p}$ value $=0.000$ ): 112 scarlet fever patients (111 gAs, $1 \mathrm{gGs}), 264$ pharyngitis patients (234 gAs, $13 \mathrm{gCs}, 17 \mathrm{gGs}$ ), 121 healthy children (93 gAs, 18 gCs, 10 gGs). Last mentioned patients mean pyogenic streptococci carriers (24.34\%).

Conclusions Carriers represents the "infection pool" for community children, maintaining persistence of source infection and explaining diminished efficacy of epidemiological measures and infection outbreaks in pediatric population.

\section{EFFECTS OF FORSYTHIA KOREANA-INCLUDED HERBAL MEDICINE TREATMENT ON UPPER RESPIRATORY TRACT INFECTION IN KOREAN CHILDREN}

doi:10.1136/archdischild-2012-302724.1738

'DY Yoo, 'SB Yang, ' JH Park, 'NG Kim, 'MA Kim, ${ }^{2} \mathrm{SY}$ Lee, ${ }^{2} \mathrm{HY}$ Choi, ${ }^{1} \mathrm{H}$ Choi. 'Department of Research and Development, Hamsoa Oriental Clinic Network; ${ }^{2}$ Department of Pharmacology and Development, Hamsoa Pharmaceutical, Seoul, Republic of Korea

Background and Aims Korea has known high antibiotics prescription in children upper respiratory tract infections (URIs) Recently, herbal therapy is be magnified instead of unnecessary antibiotics prescription. Forsythia Koreana has been extensively used for the treatment of viral and bacterial respiratory tract infections. However, it remains unclear whether the herbal medicine has beneficial effects through clinical control study in URIs. Therefore, to investigate the anti-viral effects of Forsythia Koreana included herbal medicine (FKGM) on URIs in children, we conducted a randomized, double-blind, placebo-controlled study.
Methods Participants included 29 children who received either placebo $(n=15)$ or FKGM $(n=14)$ at the onset of URIs symptoms. The therapeutic effects assessed URI symptoms scoring system given by the James A Taylor for clinical studies to identify children with a documented viral URIs. Results were evaluated using student's $t$-test; the Kaplan-Meier method was used to analyse data regarding symptom duration.

Results

Abstract 1738 Table 1 URI symptom score

\begin{tabular}{lccc}
\hline Symptom & Placebo $(\mathbf{n}=\mathbf{1 5})$ & $\mathbf{F K G M}(\mathbf{n}=\mathbf{1 4})$ & $\mathbf{p}$-value \\
\hline throat pain & $3.27 \pm 4.17$ & $1.21 \pm 2.42$ & 0.120 \\
expectoration of sputum & $4.93 \pm 4.04$ & $3.00 \pm 2.75$ & 0.146 \\
sneezing & $4.13 \pm 4.21$ & $2.21 \pm 2.52$ & 0.147 \\
runny nose & $9.60 \pm 6.67$ & $6.36 \pm 4.53$ & 0.140 \\
nasal congestion & $8.40 \pm 5.10$ & $3.79 \pm 2.72$ & $0.006^{*}$ \\
\hline
\end{tabular}

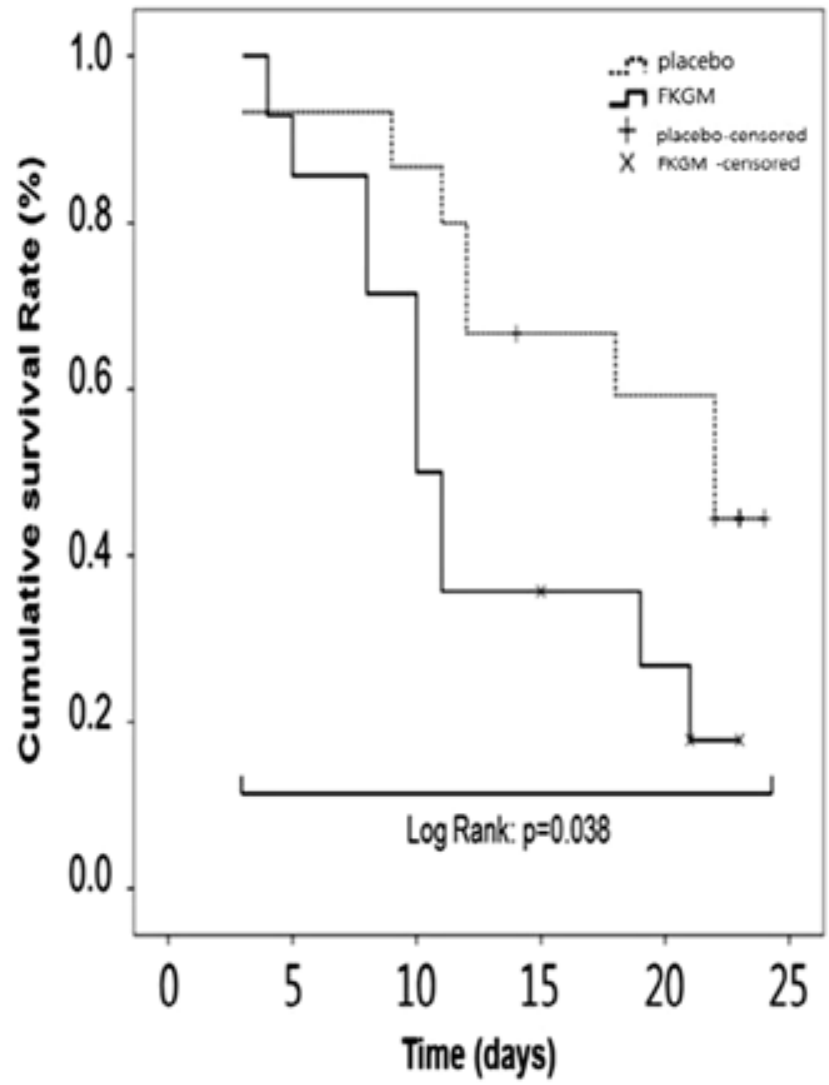

Abstract 1738 Figure 1 URI symptom duration between placebo and FKGM

Conclusions FKGM was more effective than the placebo in terms of reducing the duration of URI symptoms and reducing nasal discharge. These findings suggest that FKGM can be used for the replacement of antibiotics.

\section{SOCIOECONOMIC CHARACTERISTICS OF THE CHILDREN WHO NEEDED HOSPITALIZATION IN A PEDIATRIC INTENSIVE CARE UNIT (PICU)-RETROSPECTIVE ANALYSIS}

doi:10.1136/archdischild-2012-302724.1739

'AM Spanaki, 'M Linardakis, 'D Fitrolaki, 'T Tavladaki, 'E Blevrakis, 'S llia, 'E Vasilaki, ${ }^{1} \mathrm{E}$ Geromarkaki, ${ }^{2} \mathrm{C}$ Lionis, ${ }^{2} \mathrm{~T}$ Philalithis, ${ }^{1} \mathrm{G}$ Briassoulis. 'Pediatric Intensive Care Unit, University Hospital of Heraklion; ${ }^{2}$ Public Health and Health Care Management, Faculty of Medicine, University of Crete, Heraklion, Greece 
Background and Aims PICU provides high level of care to critically ill or injured children. Our aim was to analyse the socioeconomic characteristics of their families.

Methods 127 children, hospitalized from 10/05/2008 to 10/5/2009, were analyzed retrospectively: age, gender, parents' age, nationality, residence, marital status, number of childrens, parents' education, employment, insurance, and way of transportation, and were correlated with the days of hospitalization, severity scores and outcome. Data analysis with the SPSS 17

Results $73(57.5 \%)$ were males and 54 (42.5\%) girls, mean age 4.5 and 6.7 years respectively. $78.2 \%$ were from this country, and $4.1 \%$ tourists, $17.5 \%$ immigrants and $2.3 \%$ gypsy. No significant difference in the distribution for gender, but a statistically significant difference in educational level of both parents, in relation to nationality $(p<0,011) .75 \%$ foreign and $10 \%$ Greeks parents were primary schools, $15 \%$ and $70 \%$ high school and only $7 \%$ and $20 \%$ university graduates, respectively. Higher TISS 28 and TISS 76 (worst condition) in foreigners. Higher PRISM and PRISM Predicted Mortality (\%) severity scores (more serious condition), higher TISS 28 and TISS 76 and more days of hospitalization in children transported by ambulance/airplane. Foreigner children, transferred by ambulance or airplane, had the higher death rate.

Conclusions The severity of illness, duration of hospitalization and outcome, appear to be related to the socioeconomic characteristics of families and the way of transportation.

\section{EPIDEMIOLOGY OF SEVERE TRAUMATIC BRAIN INJURY (TBI) IN PEDIATRIC POPULATION OF THE WEST OF ALGERIA}

doi:10.1136/archdischild-2012-302724.1740

H Bouguetof, MA Negadi, K El Halimi, D Boumendil, ZC Mentouri. Pediatric Intensive Care Unit, Faculty of Medicine - Oran University, Oran, Algeria

Background and Aims Algeria is in the fourth rank of road accidents in the world.

The Aim of the study was to provide recommendations to government and health authorities, based on hard evidence, for improving health care delivery to children with severe TBI in the referral region of the university hospital center in Oran.

Method In this cohort, all children with severe TBI was admitted to the Paediatric Intensive Care Unit (PICU) of the University Hospital Centre of Oran, between the January 1, 1995 and December 31, 2007

Results The average age is 7 years $1 / 2$. Road accidents are in the origin of more than $70 \%$ of the cases in which $80 \%$ are pedestrians. $65 \%$ of pedestrians are injured on roads in rural environment (countryside). The pre hospital management is unfortunately almost absent. $1 / 3$ of children had GCS score $<5$. Median Injury Severity Scale (ISS) score was 26 (IOR 21-33; Range 9-75). The polytraumatism represents $1 / 5$ of the cases. The cerebral œedema was found in $3 / 4$ of the cases. On the 573 children, the rate of survival is $61 \%$. In this study, the road accidents represent the main cause of severe TBI in children and are responsible of a more high mortality essentially due to the absence of prehospital management.

Conclusion The improvement of prehospital management must be reduce the mortality and improve the prognosis of severe TBI and the information of the population should be promoted to reduce the frequency of severe TBI.

\section{PULSE OXIMETRY IN HEALTHY NEWBORNS AFTER MIDWIFERY SUPERVISED UNCOMPLICATED HOME BIRTHS; USE OF INTERNATIONAL ACCEPTED PERCENTILES}

doi:10.1136/archdischild-2012-302724.1741
${ }^{1} \mathrm{M}$ Smit, ${ }^{2} \mathrm{~A}$ Ganzeboom, ${ }^{3} \mathrm{~J}$ Dawson, ${ }^{2} \mathrm{AT}$ Pas. ${ }^{1}$ Obstetrics and Gynaecology: ${ }^{2}$ Neonatology, Leiden University Medical Center, Leiden, The Netherlands; ${ }^{3}$ Neonatology Research, The Royal Women's Hospital, Melbourne, VIC, Australia

Background Percentiles of oxygen saturation as a function of time from birth in uncompromised infants born at term are now defined. However, in these percentiles infants born after assisted deliveries (ventouse, forceps), by cesarean, after augmentation of labour, or epidural analgesia were also included.

Aim To evaluate if international accepted percentiles of neonatal oxygen saturation and heart rate values are applicable in infants born after non-intervention vaginal deliveries.

Methods During ten consecutive months, 27 midwives in the Leiden region used a Masimo pulse oximeter and perform measurements directly after birth infants born after non-intervention vaginal deliveries. Data was stored and analyzed using the skewness-median-coefficient of variation (LMS) method.

Results During the study period oximetry was recorded in 101 births. Percentiles of oxygen saturation and heart rate are shown in figure 1 and 2 . The percentiles are comparable to the international accepted values, except for the first 3 minutes for oxygen saturation and the first minute for heart rate.

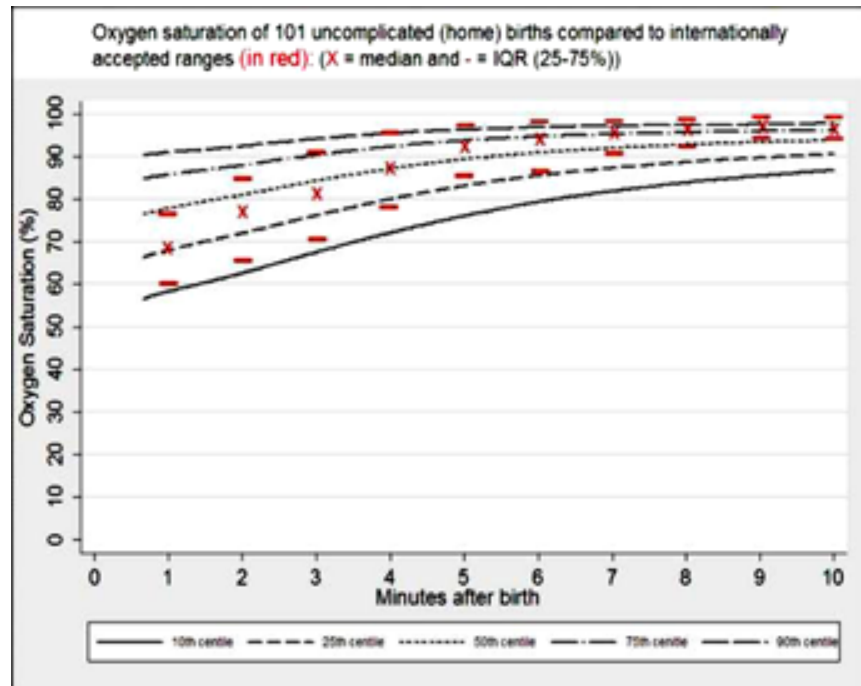

Abstract 1741 Figure 1

Neonatal heart rates of 101 uncomplicated (home) births compared to internationaly accepted ranges (in red): $(X=$ median and $-=\operatorname{IQR}(25-75 \%))$

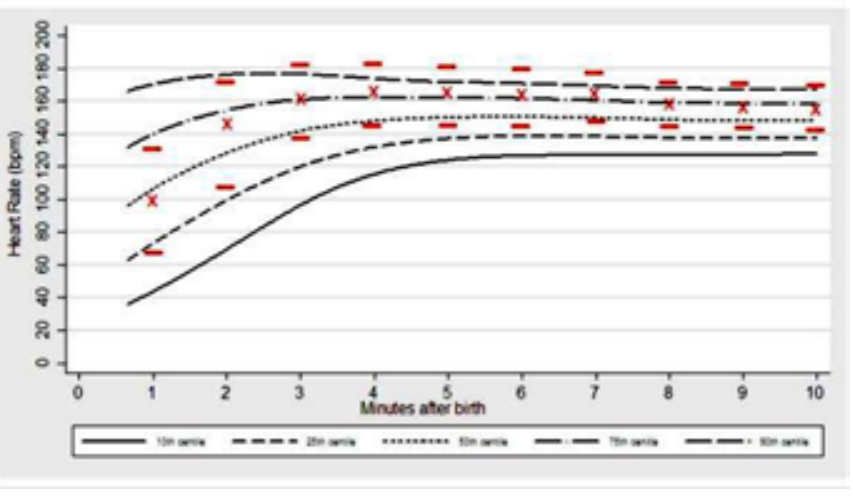

Abstract 1741 Figure 2

Conclusions The accepted percentiles for heart rate and oxygen saturation are applicable to infants born after a non-intervention 